\title{
Comments on "An economic order quantity (EOQ) for items with imperfect quality and inspection errors"
}

\author{
Hui-Ming Teng ${ }^{a}$, Ping-Hui Hsu ${ }^{b^{*}}$ and Hui Ming Wee
}

${ }^{a}$ Department of Business Administration, Chihlee Institute of Technology, Taiwan

${ }^{b}$ Department of Business Administration, De Lin Institute of Technology, Taiwan

${ }^{c}$ Department of Industrial and System Engineering, Chung Yuan Christian University, Taiwan

\begin{tabular}{l}
\hline A R T I C L E I N F O \\
\hline Article history: \\
Received 10 August 2012 \\
Accepted September 272012 \\
Available online \\
28 September 2012 \\
\hline Keywords: \\
Imperfect process \\
Misclassification errors \\
EOQ
\end{tabular}

\section{A B S T R A C T}

\begin{abstract}
The purpose of these comments is to serve as a revision to the article by Khan, Jaber, \& Bonney [2011, An economic order quantity (EOQ) for items with imperfect quality and inspection errors, International Journal of Production Economics, 133: 113-118]. This commenting paper first suggests that the revenue function derived in Khan et al. (2011) is unrealistic, and then offers revisions to complement the shortcomings.
\end{abstract}

\section{Introduction}

A Khan et al. (2011) proposed an inventory model with imperfect processes and inspection errors. Later, Hsu (2012) found a contradiction in Khan' paper between the cycle length and the holding cost per cycle, then fixed this flaw and develop a corrected EOQ. However, there are still some queries to be discussed. This commenting paper points out three queries in Khan et al.'s (2011) article that need to be re-examined. Specifically, the revenue function derived in their article is unrealistic, and thus, this commenting paper further offers corrections to complement the shortcomings. The following notation is used throughout this comment (Please refer to Khan et al.’s (2011) article).

$D$ number of units demanded per year

$y$ order size

c unit variable cost

$K$ fixed ordering cost

A a parameter used for simplifying the holding cost in Eq.(7)

* Corresponding author.

E-mail: pinghuihsu@gmail.com (P.H. Hsu)

(C) 2012 Growing Science Ltd. All rights reserved.

doi: $10.5267 / j . j$ jiec.2012.09.004 
$s$ unit selling price of a non-defective item

$v$ unit selling price of a defective item

$x$ screening rate

$d$ unit screening cost

$h$ unit holding cost

$T$ cycle length

$m_{1}$ probability of Type I error(classifying a non-defective item as defective)

$m_{2}$ probability of Type II error(classifying a defective item as non-defective)

$p$ probability that an item is defective

$t_{1}$ inspection time in a cycle

$t_{2}$ the remaining time in a cycle, after the defective items are screened out

$f(p)$ probability density function of $p$

$f\left(m_{1}\right)$ probability density function of $m_{1}$

$f\left(m_{2}\right)$ probability density function of $m_{2}$

$B_{1}$ number of items that are classified as defective in one cycle

$B_{2}$ number of defective items that are returned from the market in one cycle

$c_{a} \quad$ cost of accepting a defective item

$c_{r} \quad$ cost of rejecting a non-defective item

In Khan et al. (2011), the authors established the total profit per cycle to be written as follows:

Total profit per cycle $T P(y)$

$=$ total revenue per cycle - total cost per cycle

$=$ (the revenue from selling the good items + the revenue from selling the classified defective items)-(the procurement cost per cycle+ the screening cost per cycle

+ the holding cost per cycle).

Where the total cost per cycle is

$$
\underline{(K+c y)}+\left[\underline{d y+c_{r}} \underline{(1-p) y m_{1}}+\underline{c}_{a} \underline{p y m_{2}}\right]+h\left[\frac{\left(y-Z_{1}\right) t_{1}}{2}+Z_{1} t_{1}+\frac{Z_{2} t_{2}}{2}\right]+h\left(\frac{B_{2} T}{2}\right)
$$

with $B_{2}=y p m_{2}, B_{1}=y(1-p) m_{1}+y p\left(1-m_{2}\right), Z_{2}=Z_{1}-B_{1}, Z_{1}=y-D t_{1}$ and $t_{1}=D / x$.

And the total revenue per cycle is

$\left[\underline{s y}(1-p)\left(1-m_{1}\right)+s y p m_{2}\right]+\left[v y(1-p) m_{1}+v y p\left(1-m_{2}\right)+v y p m_{2}\right]$.

\section{Revised model}

Three queries are as follows:

1). On page 116 of Khan et al. (2011), "Figure 1 depicts the behavior of different types of inventory in the order cycle. The (red) triangle at the bottom represents the defective lot that is returned by the market and is accumulated into the salvaged lot.” However, on page 114, the (red) triangle should start at 0 , and end at $T$. The reason for this correction is that on page 115 , the author states that "the screening and consumption of the inventory continues until time $t_{1}$ ”; therefore, there would be some 
defective items that are returned from the market during this period. The revised figure is shown in Fig. 2.

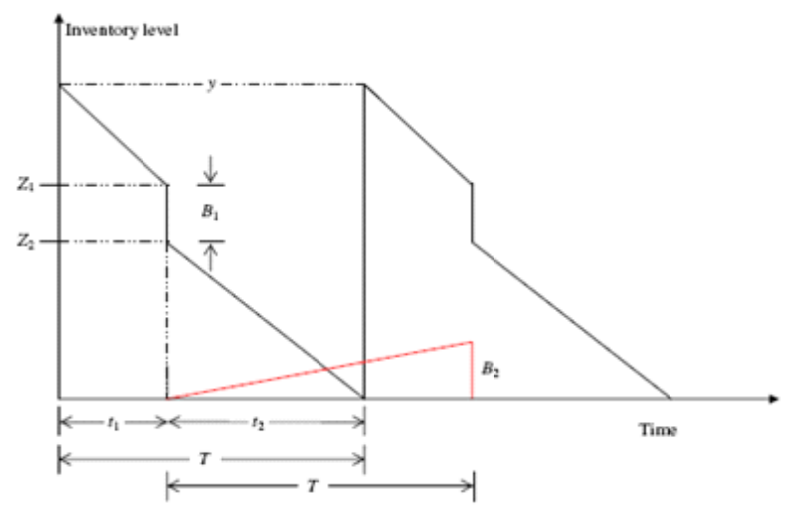

Fig. 1. Behavior of the inventory level over time (Khan et al., 2011)

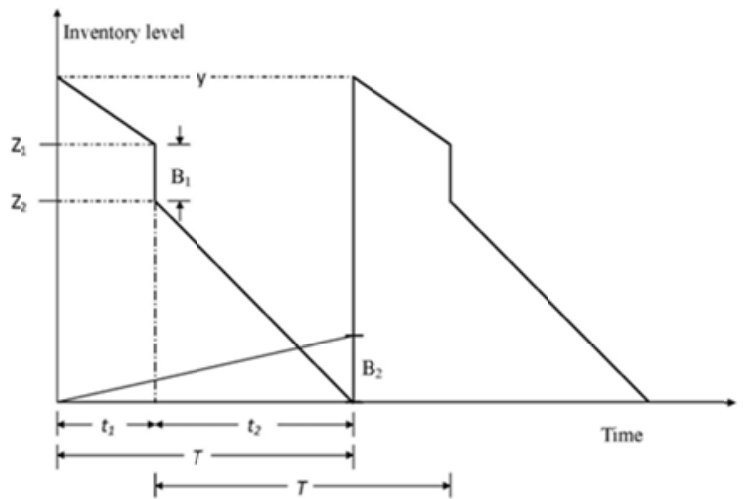

Fig. 2. Behavior of the inventory level over time (revised)

2). On page 116 of Khan et al. (2011), Sec 4: Numerical analysis, the authors defined $f(p)$ as:

$f(p)=\left\{\begin{array}{c}25,0 \leq p \leq 0.05 \\ 0, \text { otherwise }\end{array}\right.$

However, since $f(p)$ is the probability density function of random variable $p$, the following equation is needed to be satisfied:

$$
\int_{0}^{u p p e r-b o u n d} f(p) d p=1 \text {. }
$$

That is, upper-bound $=0.04$, Therefore, $f(p)$ should be defined as

$f(p)=\left\{\begin{array}{c}25,0 \leq p \leq 0.04 \\ 0, \text { otherwise } \quad, \quad \Rightarrow E(p)=0.02\end{array}\right.$

For the same reason, $f\left(m_{1}\right)$ should be defined as

$f\left(m_{1}\right)=\left\{\begin{array}{c}25,0 \leq m_{1} \leq 0.04 \\ 0, \text { otherwise } \quad, \Rightarrow E\left(m_{1}\right)=0.02\end{array}\right.$

And $f\left(m_{2}\right)$ should be defined as

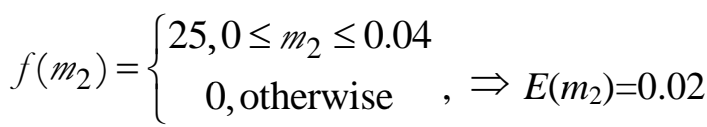

3). On page 115 of Khan et al. (2011), the revenue from selling the good items is computed as

$R_{2}=\operatorname{sy}(1-p)\left(1-m_{1}\right)+\operatorname{sypm}_{2}$.

However, the amount of $\mathrm{ypm}_{2}$ (which are defective items but classified as non-defective) returned from the market. In other words, this amount is counted as defective item instead of non-defective. Therefore, the total revenue per cycle should be

As a result, the total revenue should be rewritten as

$\operatorname{sy}(1-p)\left(1-m_{1}\right)+v y(1-p) m_{1}+v y p$ 
From Eq.(6), the total profit per cycle can now be written as

$$
\begin{gathered}
T P(y)=\left[\operatorname{sy}(1-p)\left(1-m_{1}\right)\right]+\left[v y(1-p) m_{1}+v y p\right]-\left\{K+c y+d y+c_{r}(1-p) y m_{1}+c_{a} p y m_{2}+h\left[\frac{\left(y-Z_{1}\right) t_{1}}{2}+Z_{1} t_{1}\right.\right. \\
\left.\left.+\frac{Z_{2} t_{2}}{2}\right]+h\left(\frac{B_{2} T}{2}\right)\right\}=\left[\operatorname{sy}(1-p)\left(1-m_{1}\right)\right]+\left[v y(1-p) m_{1}+v y p\right]-\left\{K+c y+d y+\operatorname{cr}(1-p) y_{1}+\operatorname{capym}_{2}+\frac{h}{2}\left[\left(\frac{2}{x}-\frac{D}{x^{2}}+\frac{A^{2}}{D}\right) y^{2}+y p m_{2} T\right]\right\}
\end{gathered}
$$

where $A=1-D / x-\left(m_{1}+p\right)+p\left(m_{1}+m_{2}\right)$.

From Eq.(7), with $T=\frac{y(1-p)\left(1-m_{1}\right)}{D}$ and $E[T]=\frac{y(1-E[p])\left(1-E\left[m_{1}\right]\right)}{D}$, the expected total profit should be revised as

$$
\begin{aligned}
& E[T P U(y)]=\frac{E[T P(y)]}{E[T]}=s D+\frac{v D E\left[m_{1}\right]}{\left(1-E\left[m_{1}\right]\right)}+\frac{v D E[p]}{(1-E[p])\left(1-E\left[m_{1}\right]\right)} \\
& -\frac{D\left[\frac{K}{y}+c+d+c_{r}(1-E[p]) E\left[m_{1}\right]+c_{a} E[p] E\left[m_{2}\right]+\frac{h}{2}\left\{\left(\frac{2}{x}-\frac{D}{x^{2}}+\frac{E\left[A^{2}\right]}{D}\right) y\right\}\right]}{(1-E[p])\left(1-E\left[m_{1}\right]\right)}-h \frac{y E[p] E\left[m_{2}\right]}{2} .
\end{aligned}
$$

Therefore, the first derivative of Eq. (8) is the same as Khan et al. (2011), and the optimal order size as well.

\section{Numerical example}

Using the same data from Khan et al. (2011): $D=50$ 000units/year, $c=\$ 25 /$ unit, $K=\$ 100 /$ cycle, $s=$ \$50/unit, $v=\$ 20 /$ unit, $x=1$ unit/min ( $x=1(60)(8)(365)=175200$ units), $d=\$ 0.5 /$ unit, $h=\$ 5 /$ unit, $c_{a}=$ $\$ 500 /$ unit, $c_{r}=\$ 100 /$ unit, $E(p)=0.02, E\left(m_{1}\right)=0.02, E\left(m_{2}\right)=0.02$, and $E\left(A^{2}\right)=0.456$, the optimal values of the order size and the annual profit are as $y^{*}=1455$ units, and ETPU $=\$ 1094050$ /year (Khan et al.'s ETPU $=\$ 1095090 /$ year, the error is $\$ 1041 /$ year $)$.

\section{Conclusion}

This commenting paper points out three queries in Khan et al.'s (2011) article; specifically, the revenue function derived in their article is unrealistic. This commenting paper further offers corrections to these shortcomings with a revised model. The numerical example is demonstrated for comparison.

\section{Acknowledgment}

The authors wish to express their deep appreciation to the National Science Council and De Lin Institute in Taiwan for their financial support. They would also like to thank the editor and reviewers for their valuable comments.

\section{References}

Hsu, L.F. (2012). A note on "An economic order quantity (EOQ) for items with imperfect quality and inspection errors". International Journal of Industrial Engineering Computations, 3(5), 939-948.

Khan, M., Jaber, M.Y., \& Bonney, M. (2011). An economic order quantity (EOQ) for items with imperfect quality and inspection errors. International Journal of Production Economics, 133, 13118. 